\title{
Synchrotron infrared transmission spectroscopy of a quantum cascade laser correlated to gain models
}

\author{
Peter Friedli, ${ }^{1}$ Hans Sigg, ${ }^{1}$ Andreas Wittmann, ${ }^{2}$ Romain Terazzi, ${ }^{2}$ Mattias Beck, ${ }^{2}$ \\ Andrzej Kolek, ${ }^{3}$ and Jérôme Faist ${ }^{2}$ \\ ${ }^{1}$ Laboratory for Micro- and Nanotechnology, Paul Scherrer Institut, CH-5232 Villigen PSI, Switzerland \\ ${ }^{2}$ Institute for Quantum Electronics, ETH Zürich, CH-8093 Zürich, Switzerland \\ ${ }^{3}$ Department of Electronics Fundamentals, Rzeszow University of Technology, Rzeszow 35-959, Poland
}

(Received 5 October 2012; accepted 20 December 2012; published online 10 January 2013)

\begin{abstract}
We measure the broadband optical gain and absorption spectra between 0.1 and $0.7 \mathrm{eV}$ in a two-phonon resonance design quantum cascade laser, with the infrared beam of a synchrotron light source, and correlate them to established simulation models, based on the density matrix formalism, and on the non-equilibrium Green's function theory. We show that accounting for the distribution of carriers in momentum space improves the description of the high-energy absorption from the excited states located close to the active wells and results in an accurate prediction of the gain. (C) 2013 American Institute of Physics. [http://dx.doi.org/10.1063/1.4774319]
\end{abstract}

Despite the impressive advances made in quantum cascade laser $(\mathrm{QCL})^{1}$ research over the last decade concerning wavelength coverage, gain bandwidth, emission efficiency as well as operation temperature, ${ }^{2}$ the extent of quantitative methods to assess the device's main optical properties-gain and loss-are still underdeveloped. There is, therefore, a need for methods that deliver significant parameters for benchmarking models and simulations, such as Monte Carlo, density matrix (DM) formalism, and non-equilibrium Green's function (NEGF) theory.

Well-known experimental methods to determine overall gain and loss involve measuring the threshold current densities for different cavity lengths or facet reflectivities of the same QCL design. Another possibility is the measurement of the contrast of the Fabry-Pérot (FP) fringes in the subthreshold electroluminescence (EL) as a function of injected current. ${ }^{3}$ However, these techniques are inherently limited to the spectral region around the radiative transition. Measurements using a multi-section cavity technique ${ }^{4}$ overcome this limitation, but require elaborately processed devices. Alternatively, the coupling of broadband infrared (IR) light from a Fourier transform infrared (FTIR) spectrometer into a QCL device was shown to be a direct and simple method for characterization of broad-area waveguide QCL devices in continuous wave $(\mathrm{CW})$ operation. ${ }^{5}$ However, to enable studies of high performance single-mode (SM) QCL devices or for investigations requiring pulsed operation, the sensitivity of such methods need to be improved. These problems can be overcome using time domain spectroscopy, ${ }^{6}$ where fs-long laser pulses are used to create intense broadband pulses in the mid-IR. Another option, as employed in this work, is to use a broadband, high brightness IR beam of a synchrotron source $^{7}$ for transmission studies. Here, the intensity of the diffraction-limited spot size is typically $100-1000$ higher than from a globar source, ${ }^{8}$ which allows to achieve a high signal-to-noise ratio for transmission measurements on single transverse mode mid-IR laser waveguides.

In this paper, we systematically compare two well-known models, applied to a particular QCL design, to synchrotron based broadband measurements. The two models go beyond semi-classical models and are based on a DM formalism, ${ }^{9,10}$ and on the NEGF, ${ }^{11,12}$ respectively. While the first method is less computationally intensive, the latter is more powerful and, in our case, includes scattering in $k$-space. ${ }^{13}$ Nevertheless, in order to keep the numerical load of the NEGF within reasonable limits, the space-, energy-, and momentumresolution is decreased, requiring a careful adaption of the input digitized potential to mimic the true structure. Details of the DM simulation, which, in our case, assumes the electron temperature is the lattice temperature, and on the used scattering mechanisms, which are of the interface roughness, LOphonons, alloy disorder, and ionized impurities types, are shown in Ref. 10. For the case of the NEGF approach, we use a real-space basis ${ }^{12}$ with $1 \mathrm{~nm}$ grid spacing and a $2 \mathrm{D}$ mesh uniformly spaced both in energy and $k^{2}$. Boundary conditions and details of numerical procedure are described in Refs. 13 and 14 . The only temperature that enters this model is the lattice temperature, however, highly non-thermal carrier distributions were observed in the lower active wells subbands. The material gain in NEGF model was calculated using Coulomb gauge and neglecting terms with $\delta \Sigma .{ }^{11}$ Contributions to gain from the transitions at different $k$ 's were calculated individually and then summed up to the total gain. Scattering mechanisms included in NEGF calculations are LO-phonon, interface roughness, alloy disorder, ionized impurity, and LAphonon, as explained in detail in Ref. 13, and non-parabolicity was taken into account as well.

We study the case of a high-performance QCL based on the two-phonon resonance $(2 \mathrm{Ph})$ design. ${ }^{15}$ The designed band structure and electron states are given in Fig. 1(a). The energy levels and the relevant dipole-matrix elements were calculated using the self-consistent solution of the Poisson and Schrödinger equations, employing an energy-dependent effective mass, which accounts for non-parabolicity. Light emission in the sub-threshold regime occurs from the upper laser state $|12\rangle$ down to states $|8\rangle-|10\rangle$, and finally concentrates on the main lasing transition $|12\rangle \rightarrow|10\rangle$ at $8.4 \mu \mathrm{m}$ for increased biases. 

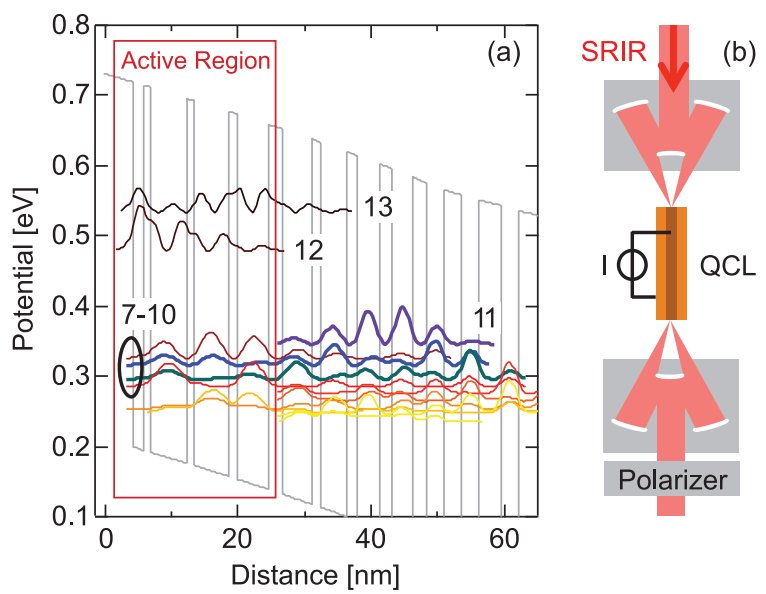

FIG. 1. (a) Conduction band diagram and moduli squared of the relevant electron wavefunctions at an applied field of $33 \mathrm{kV} / \mathrm{cm}$. Part of the injector $(8 \mathrm{~nm})$ is Si-doped to $1.5 \times 10^{17} \mathrm{~cm}^{-3}$. The states on the right side of the active region, that are drawn in bold lines, give rise to the increased highenergy absorption. (b) Sketch of the transmission measurement setup, showing the $15 \times$ amplification dispersion-less Schwarzschild lenses.

For the measurement, the devices were processed into narrow-ridge low-loss heterostructure waveguides (width $13.9 \mu \mathrm{m}$ ), buried in iron-doped InP. The active device was cleaved to $3 \mathrm{~mm}$ long bars, which is the best compromise between effectively suppressing stray light and ensuring a detectable transmission for the unbiased device, i.e., when the absorption in transverse-magnetic (TM) polarization is strong. Only in TM polarization, where the electric field is polarized along the growth direction of the structure, selection rules are fulfilled and the electric field can couple to the intersubband transition, while the transverse-electric (TE) polarized light is not affected. ${ }^{16}$ The IR light is then focused onto the waveguide facet of the device in a microscope, consisting of Schwarzschild lenses to avoid any dispersion, c.f. Fig. 1(b), and the transmission is recorded with a FTIR spectrometer. The normalized sub-threshold transmission spectra $t=\frac{T}{T_{0}}$, which is the measured transmission $T$ through the device with respect to the transmission $T_{0}$ through a predefined $15 \times 8 \mu \mathrm{m}^{2}$ aperture, when entrance and exit optics are in coincidence, are given for both TM- and TE-polarized light in Fig. 2(a) at an effective device temperature of $349 \mathrm{~K}$, from no bias up to a current of $I=0.97 \cdot I_{t h}$, where $I_{t h}$ defines the threshold current for lasing operation. The effective device temperature is kept constant by monitoring the InGaAs interband absorption cutoff at around $700 \mathrm{meV} .{ }^{5}$ We model the peak transmission $t$ with a multi-pass FP model $t$ $=c \cdot(1-r)^{2} \cdot e^{-\alpha l} \cdot \frac{1}{1-r^{2} \cdot e^{-2 \alpha l}}$, where the coupling coefficient $c$ accounts for the normalization to the reference aperture measurement, $r=0.27$ is the mirror reflectivity at the cleaved facets, and $\alpha$ is the total absorption over the device length $l$. We define the total absorption by $\alpha=\alpha_{0}-g_{m} \cdot I$, where $\alpha_{0}$ is the total waveguide loss and $g_{m}$ is the modal gain coefficient. We then fit $t$ for the parameters $c, \alpha_{0}$, and $g_{m}$, which gives excellent agreement to the data, as shown in Fig. 2(b). This strongly supports our assumption of a linear dependence of the gain on the injection current. Furthermore, the extrapolated threshold currents $I_{t h}$ fit very well to the measured ones. Also, good agreement is obtained between $I_{t h}$ in CW mode at a distinct effective device temperature $T_{D}$,
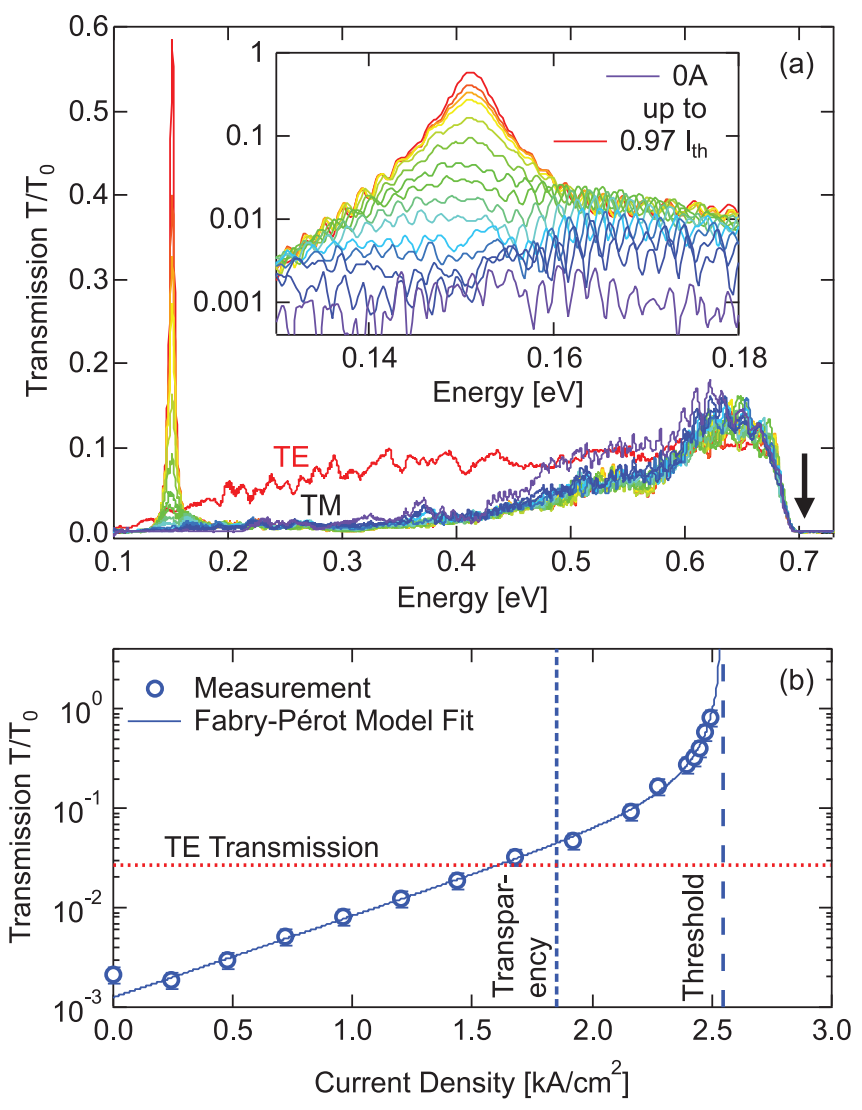

FIG. 2. (a) Transmission spectra for TM and TE polarization measured for increasing biases of $0-32.8 \mathrm{kV} / \mathrm{cm}$ (up to $97 \%$ of the threshold current), while maintaining the effective device temperature $(349 \mathrm{~K})$ by monitoring the bandgap onset (vertical arrow). Inset: Transmission spectra near the peak gain of $145 \mathrm{meV}$ shown on a log-scale. (b) Current dependent peak transmissions for the effective device temperature of $349 \mathrm{~K}$ together with the result of the three-parameter fit of the Fabry-Pérot transmission model (full line). Vertical dashed lines indicate the obtained values for transparency and threshold currents.

and the respective $I_{t h}$ measured under low duty-cycle pulsed conditions with the heat sink temperature set to $T_{D}$. We determine a material gain coefficient of $(8.6 \pm 0.2) \mathrm{cm} / \mathrm{kA}$, where an overlap factor $\Gamma$ of 0.73 , determined by $2 \mathrm{D}$ ComsoL simulations using the measured lateral dimensions, is taken into account. This value is comparable to the measured values for similar devices ${ }^{17}$ and matches reasonably well to the $7.9 \mathrm{~cm} / \mathrm{kA}$ from NEGF simulations. As shown in Fig. 3(a), the prediction by the DM model (equal to $6.5 \mathrm{~cm} / \mathrm{kA}$ ) is considerably lower. However, as the same model also underestimates the transparency current, which is the current at where the intersubband gain compensates the total waveguide losses, the predicted values of threshold are close to the experimental ones, as pointed out in Ref. 10. The current-independent total waveguide loss, defined as $\alpha_{0}=\alpha_{W G \text {,empty }}+\Gamma \cdot \alpha_{I S B}$, where $\alpha_{W G, \text { empty }}$ arises from the free carrier absorption in the doped cladding layers, and $\alpha_{I S B}$ are losses from intersubband transitions at zero bias, ${ }^{17}$ is found to be $(11.7 \pm 0.3) \mathrm{cm}^{-1}$. From the determined coupling coefficient of up to $10 \%$ and the spectrally integrated power transmitted through a $10 \mu \mathrm{m}$ pinhole, we expect roughly 0.1 to $1 \mu \mathrm{W}$ to be coupled into the waveguide.

For a complete assessment of the models, we extract the absorption spectra $\alpha(\omega)$ from the transmission spectra $T(\omega)$. This, however, needs some special attention, because such a 

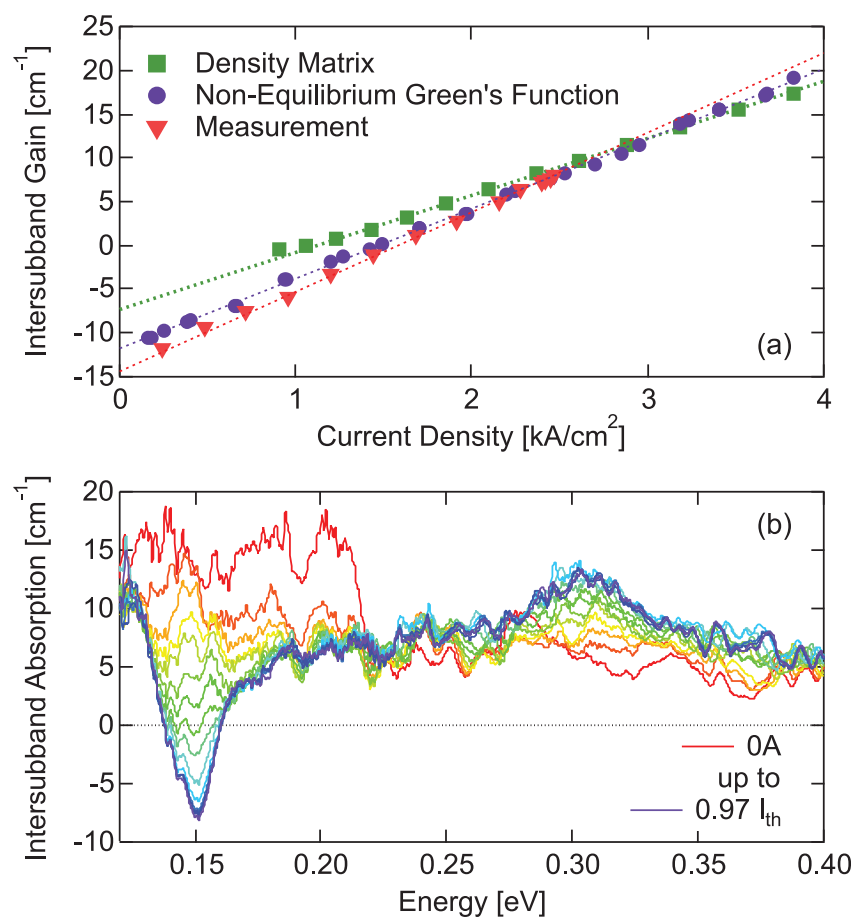

FIG. 3. (a) Plot of the intersubband peak gain as a function of current density, comparing the measurements with the prediction of the nonequilibrium Green's function and density matrix simulations. (b) Pure intersubband absorption as determined by the ratio of TM and TE transmission and by taking into account multiple passes through the cavity.

conversion to $\alpha(\omega)$ requires knowledge of the coupling $c(\omega)$ at all frequencies, which is not directly accessible from this measurement. However, in parallel studies of slightly smaller (width $\sim 10 \mu \mathrm{m}$ ) but undoped waveguides, where TM absorption is negligible, we determined a comparable coupling of both TM and TE-polarized light for energies above the peak gain energy. Also, the obtained TE absorption value at resonance $(0.145 \mathrm{eV})$ of $2 \mathrm{~cm}^{-1}$ for the determined TM-coupling seems an accurate measure of the nonresonant waveguide losses. As TM and TE waveguide losses are expected to be similar, ${ }^{18}$ we normalize the wavelength dependent coupling by taking the TE transmission as a reference. In this way, we can extract the pure intersubband losses and gain for higher energies from the TM transmission, as given in Fig. 3(b).

In Fig. 4, we correlate the broadband spectrally resolved measurement at $32 \mathrm{kV} / \mathrm{cm}$ to the simulated intersubband absorption for both models at an applied field of $32 \mathrm{kV} / \mathrm{cm}$ at $349 \mathrm{~K}$. For the models, we see a distinct difference in the energy range from 0.2 to $0.32 \mathrm{eV}$, while above this there is a close match. The reason for the higher absorption in the NEGF approach is the higher occupation of those injector states adjacent to the active wells as highlighted in Fig. 4, which penetrate the active region and are in resonance with the lower laser states for high k-values. In Ref. 13, it was shown that these states are populated from lower laser subbands by currents flowing at low and high- $k$ values. Therefore, as shown in the lower panel of Fig. 4, the $k$-resolved intersubband absorption summed up to a given $k$-value continues to increase up to these high $k$-values. This absorption cannot be predicted by the DM model, which assumes a thermal distribution in the subband equal to the lattice one.

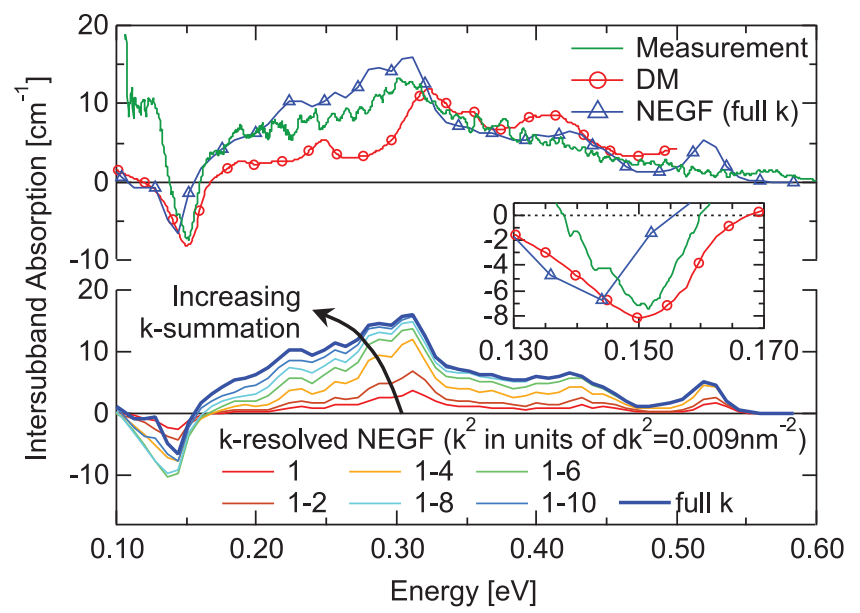

FIG. 4. Top panel: Comparison of the modeled and the measured absorption (scaled by the overlap factor) at a bias of $32 \mathrm{kV} / \mathrm{cm}$. The main difference lies in the range 0.2 to $0.3 \mathrm{eV}$, where the NEGF model matches the higher absorption better. Inset: The DM approach reproduces the gain peak position accurately. Bottom panel: $k$-resolved absorption for the NEGF model at an applied field of $32 \mathrm{kV} / \mathrm{cm}$, given in units of $d k^{2}=0.009 \mathrm{~nm}^{-2}$. The absorption strength heavily depends on the amount of $k$-states included, which is marked by an arrow.

Interestingly, the use of an approximation where the electron temperature is still assumed to be constant between all states, but satisfying a kinetic balance, does not significantly improve the fit in the frequency range $0.15-0.3 \mathrm{eV}$ while strongly underestimating the gain, as already discussed in Ref. 9. This is because the DM, in contrast to the NEGF approach, does not allow for non-uniform heating of laser subbands and, hence, cannot redistribute carriers neither in $k$-space nor in z-space between active wells and the injector.

As expected, the DM model better predicts the energy levels and results in a more accurate gain peak location, because it is not bound to a discrete mesh. In the case of the NEGF model, this could be improved by allowing for a finer mesh and/or a more accurate adaption of the well depths; this however at the drawback of high computational time. Finally, we point-out that including terms with $\delta \Sigma$ when calculating material gain in the NEGF model (as in the full theory of Ref. 11) mostly gives changes around the gain peak position, and only slightly changes the absorption for higher energies.

In summary, the potential for synchrotron based transmission studies is demonstrated on a narrow-waveguide QCL device. The evaluation of the transmission close to threshold enables us to extract the intersubband gain and losses both quantitatively and spectrally. We showed that high $k$-absorption needs to be taken into account to adequately model the absorption and gain features in quantum well structures, and that the NEGF simulations with $k$-space resolution reproduce best the overall data.

We would like to thank Dr. Philippe Lerch and Dr. Lee Carroll for beamline support, and Stefan Stutz for technical support. Experiments were performed at the X01DC beamline of the Swiss Light Source. The Swiss National Science Foundation (SNSF) funded part of this work. A.K. acknowledges support from Polish National Centre for Research and Development (1PBS B-179616) and ETH Zurich (hospitality). 
${ }^{1}$ J. Faist, F. Capasso, D. L. Sivco, C. Sirtori, A. Hutchinson, and A. Y. Cho, Science 264(5158), 553-556 (1994).

${ }^{2}$ A. Müller and J. Faist, Nat. Photonics 4(5), 291 (2010).

${ }^{3}$ D. Hofstetter and J. Faist, IEEE Photonics Technol. Lett. 11(11), 1372 1374 (1999).

${ }^{4}$ T. Gresch, J. Faist, and M. Giovannini, Appl. Phys. Lett. 94, 161114 (2009).

${ }^{5}$ D. G. Revin, L. Wilson, J. W. Cockburn, and A. Krysa, Appl. Phys. Lett. 88(13), 131105 (2006).

${ }^{6}$ W. Parz, T. Müller, J. Darmo, K. Unterrainer, M. Austerer, G. Strasser, L. Wilson, J. W. Cockburn, A. Krysa, and J. Roberts, Appl. Phys. Lett. 93, 091105 (2008).

${ }^{7}$ P. Lerch, L. Quaroni, J. Wambach, J. Schneider, D. B. Armstrong, D. Rossetti, F. L. Mueller, P. Peier, V. Schlott, L. Carroll, P. Friedli, H. Sigg, S. Stutz, and M. Tran, J. Phys.: Conf. Ser. 359, 012003 (2012).

${ }^{8}$ E. Levenson, P. Lerch, and M. C. Martin, Infrared Phys. Technol. 49(1), 45-52 (2006).
${ }^{9}$ R. C. Iotti and F. Rossi, Rep. Prog. Phys. 68(11), 2533-2571 (2005).

${ }^{10}$ R. Terazzi and J. Faist, New J. Phys. 12(3), 033045 (2010).

${ }^{11}$ A. Wacker, Phys. Rev. B 66(8), 085326 (2002).

${ }^{12}$ T. Kubis, C. Yeh, and P. Vogl, Phys. Status Solidi C 5(1), 232-235 (2008).

${ }^{13}$ A. Kolek, G. Hałdaś, and M. Bugajski, Appl. Phys. Lett. 101(6), 061110 (2012).

${ }^{14}$ G. Haldas, A. Kolek, and I. Tralle, IEEE J. Quantum Electron. 47(6), 878885 (2011).

${ }^{15}$ A. Wittmann, Y. Bonetti, J. Faist, E. Gini, and M. Giovannini, Appl. Phys. Lett. 93(14), 141103 (2008).

${ }^{16}$ H. C. Liu, M. Buchanan, and Z. R. Wasilewski, Appl. Phys. Lett. 72(14), 1682-1684 (1998).

${ }^{17}$ A. Wittmann, T. Gresch, E. Gini, L. Hvozdara, N. Hoyler, M. Giovannini, and J. Faist, IEEE J. Quantum Electron. 44(1), 36-40 (2008).

${ }^{18}$ D. G. Revin, J. W. Cockburn, S. Menzel, Q. Yang, C. Manz, and J. Wagner, J. Appl. Phys. 103, 043106 (2008). 